\title{
A study of shading and resistive loss from the fingers of encapsulated solar cells
}

\author{
M.F. Stuckings*, A.W. Blakers
}

Department of Engineering, Centre for Sustainable Energy Systems, Faculty of Engineering and Information Technology, The Australian National University, ACT 0200 Australia

Received 15 December 1998; received in revised form 10 March 1999

\begin{abstract}
Optimised solar cell design is dependent on the assumed shading and resistance losses associated with front contacts. In this study, a spectrophotometer with integrating sphere attachment was used to measure the reflection from the front surface of encapsulated silver electroplated front contact solar cells. The results obtained are in good agreement with a previous study by one of the authors using a different method. The measured effective shading loss is about one third of the coverage fraction of the cell grid because of trapping of light reflected from the grid. The grid loss in $4 \times 5 \mathrm{~cm}$ silver electroplated front contact solar cells was found to be similar to the predicted loss from buried grid and rear point contact solar cells operating at 30 suns concentration. (C) 1999 Elsevier Science B.V. All rights reserved.
\end{abstract}

Keywords: Optical losses; Encapsulation; Concentrator; Contacts

\section{Introduction}

In a conventional silicon solar cell, metal fingers are placed on the front surface to draw current from the cell. Three major losses associated with these fingers are:

1. Optical loss, due to reflection or absorption of incident light by the fingers.

2. Finger resistance, due to ohmic resistance in the metal fingers.

3. Lateral spreading resistance, due to the finite resistance of the silicon as carriers move from the point of generation to the contacts.

* Corresponding author. Tel.: + 61-2-6249-5132; fax: + 61-2-6249-0506.

E-mail address: mick@faceng.anu.edu.au (M.F. Stuckings) 


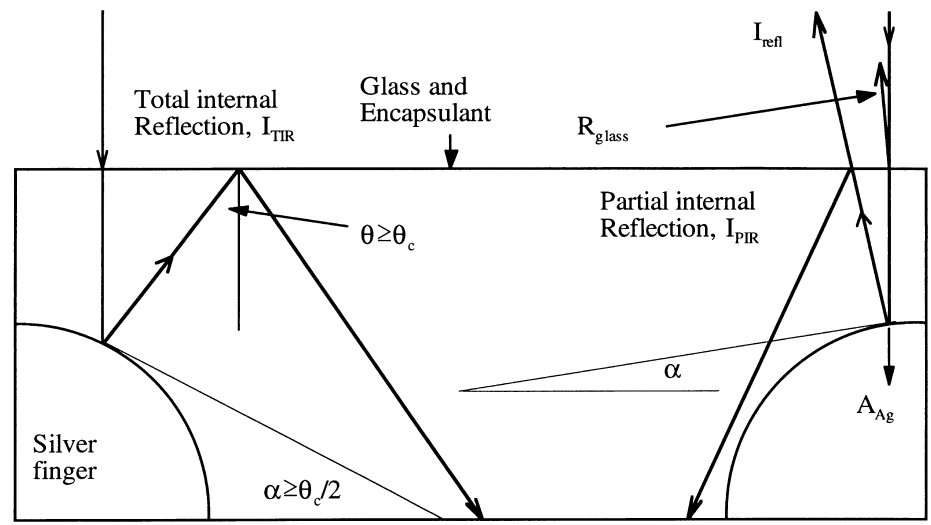

Fig. 1. Definition of important parameters.

Other losses include contact recombination and metal-silicon contact resistance.

This paper analyses the first three losses. It is important to know accurately the optical loss from the metal fingers in the encapsulated solar cell since most solar cells will be encapsulated before use in the field.

For specularly reflective fingers with a rectangular cross-section the reflection/absorption loss will be equal to the ratio of the geometric width of the fingers to the finger spacing. For fingers with a curved cross section, some of the reflected light will be internally reflected at the air glass interface as depicted in Fig. 1. Any light deflected by more than the critical angle will be totally internally reflected. The result is that the effective optical width of the fingers is less than the geometric width.

Previous work by Blakers [1] demonstrated that the effective shading fraction of encapsulated silver plated front contacts is less than $36 \%$ of the measured geometric width for light close to normal incidence. The work by Blakers was based on first-order calculations and measurements of $I_{\mathrm{sc}}$ versus plating width, using isopropanol to simulate encapsulation.

The experimental work presented here consists of measurements of absolute diffuse reflectance from samples of different plating widths and surface texture.

\section{Cell design}

The effective width of the contact fingers is an important parameter in the optimisation of solar cell design. In one sun cell design the typical geometric coverage fraction is about $4 \%$ and in this case the effect of semi-circular cross-section fingers would be to reduce the shading loss to approximately $1.5 \%$. For front contact concentrator cells operating at 30 suns, the geometric coverage fraction is $\sim 10-15 \%$ and the effect of an effective width of 0.36 is a reduction in shading loss to $3-5 \%$. 
For a number of mid-concentration systems under development at the moment, e.g. Entech [4], Euclides [5] and the ANU PV/trough system [6], the cell width is approximately $4 \mathrm{~cm}$. The competing design technologies are interdigitated back contact and three types of front contact cell: photolithographically defined electroplated contacts, laser defined (or buried) plated contacts and screen printed contacts.

The front contact schemes can all be combined with a prismatic cover (e.g. Ref. [4]) or a sculpted internal reflection cover [7] to divert light away from the contacts. In the case of the screen printed cells, one of these two schemes will be required. These technologies have yet to demonstrate long term reliability in a reflective optics system, where the receiver is exposed to the elements. It is also not clear whether the internal reflection cover will prove cost-effective, as it replaces cheaper float glass with a more costly product, and requires optical alignment, which is difficult to achieve in a full receiver module. If a deflecting technology is used, the photolithographic and laser grooved technologies become very similar in shading loss. The photolithographic design has a significant advantage over the other two approaches in that the siliconmetal contact area is much smaller, which reduces contact recombination losses. These can be significant in a concentrator cell because of the large number of fingers required.

\section{Experimental results}

Solar cells designed for operation under 30 suns concentration were fabricated on randomly textured and polished silicon wafers. Metal fingers were formed by silver electroplating. The initial width of the fingers was typically $6-8 \mu \mathrm{m}$ and the postplating width was $30-46 \mu \mathrm{m}$. Finger spacing was 200 or $400 \mu \mathrm{m}$ giving a geometric coverage fraction of $9-18 \%$.

The cross-section of the fingers after plating was observed by SEM to be roughly a half circle (see Fig. 2). The reason for this is that the silver plating occurs approximately equally in all directions and so provided that the plating thickness is several times the starting width a half circular cross section results. In actual fact the fingers usually grow slightly faster in the vertical direction than the horizontal direction, probably due to the greater rate of plating solution interchange at the apex of the growing finger than next to the silicon surface. The finger is dimpled on a small $(<1 \mu \mathrm{m})$ scale. The cells were encapsulated using clear silicone rubber pottant $\left(n_{i}=1.404\right)$ and $1 \mathrm{~mm}$ low iron glass $\left(n_{i}=1.511\right)$. The absorption in the encapsulant package was measured to be less than $1 \%$ and the reflection at the glass/silicone interface was calculated to be $0.1 \%$.

The reflection of normally incident light from encapsulated and unencapsulated silicon solar cells was measured using a Cary 5 spectrophotometer with a Diffuse Reflectance Accessory (an integrating sphere). Reference reflection from polished and textured silicon without fingers was also measured for both unencapsulated and encapsulated cells.

The integrating sphere is designed to accept samples flush with the outer surface of the sphere. For samples with an angular reflectance distribution different from the 


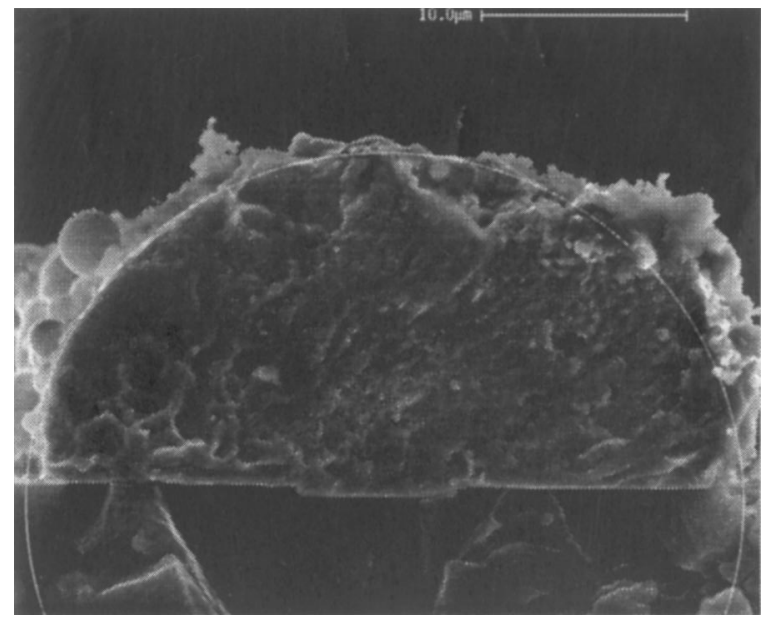

Fig. 2. The cross-section of a silver electroplated finger. The finger shows some end damage and spattering due to laser cutting. The cross section is approximately circular. A circle is included as a reference.

reflectance standard this can introduce errors due to the different flux impinging on the walls of the entrance port. It was found necessary to use samples which were coincident with the inner surface of the integrating sphere.

The reflectance of electroplated silver was measured to be $97 \%$ with $3 \%$ absorption loss. Approximately $20 \%$ of the reflectance is diffuse rather than specular. This is due to the small scale surface roughness mentioned above. The $3 \%$ absorption by the silver fingers directly adds to the loss due to the fingers and is added to the effective width. The actual absorbance could be slightly less than the measured value since reflectance increases with incident angle.

The reflectance measurements for encapsulated samples are presented in Fig. 3. The optical loss due to the fingers is calculated by subtracting the reflectance of the samples without contact fingers from the reflectance of the samples with silver electroplated fingers. To this is added the $3 \%$ loss due to absorption by the fingers. The effective width is defined as the ratio of the measured optical loss to that which would be expected for flat fingers for which all light striking the fingers is lost. For polished samples the effective width is about $29 \%$ at a geometric coverage fraction of $10 \%$ rising to about $36 \%$ at $18 \%$ coverage fraction. For the textured samples the effective width is about $32 \%$, and this would likely rise to approximately $38 \%$ at $18 \%$ coverage fraction.

In these measurements, it is implicitly assumed that the geometric coverage fraction is small enough that the probability of the light striking a second finger is small. In this case light is unlikely to strike a silver finger and be diverted out of the encapsulant. Since the geometric coverage fraction of the fingers of the concentrator solar cells used in this study is quite high (9-18\%), the probability of striking a second finger is not negligible. In this case the effective width will be a function of the geometric shading fraction and the effective width of an isolated finger. This gives rise to the small 


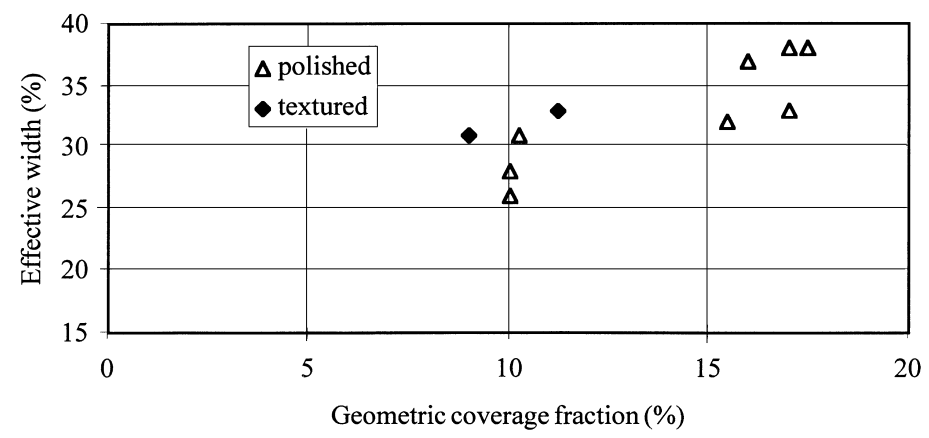

Fig. 3. Measured effective width versus geometric coverage fraction for encapsulated electroplated silver fingers.

increase in effective width with increased geometric coverage fraction apparent in Fig. 3. Due to the low reflectance of the textured surface, light would on average only make one internal reflection before being coupled into the silicon, compared with typically two bounces in the case of a polished surface, and would therefore have a lower probability of striking a finger and being deflected out. It would thus be expected that the increase in effective width with coverage fraction would be less for textured wafers than for polished wafers, although this study does not confirm this.

Approximately $20 \%$ of the reflectance is "diffuse". It would be expected that the diffuse reflectance would decrease the net reflectance, due to scattering into the third dimension (i.e. along the finger length) which increases the angle of incidence at the front surface.

With random texturing the fingers lie on textured silicon and this appears to slightly increase the effective width. In Ref. [1], all samples had the fingers located on polished areas, even if the remainder of the surface was textured, and no difference was noted between textured and untextured wafers. The feature size of the random pyramid texturing used in this work was about $10-15 \mu \mathrm{m}$. Making the pyramids much smaller than the finger width would probably reduce the reflectance to a level similar to the polished samples. Fig. 4 shows an electron microscope picture of electroplated fingers on a textured surface. Fig. 5 is a section of an electroplated finger on a polished surface for comparison.

\section{Numerical calculations}

The expected excess reflection loss from the fingers was calculated based on the following assumptions:

1. The refractive indices are independent of wavelength.

2. The fingers have a semi-circular cross-section (see Fig. 2).

3. The silver fingers are $97 \%$ reflective $\left(R_{\mathrm{Ag}}=0.97, A_{\mathrm{ag}}=0.03\right)$. 


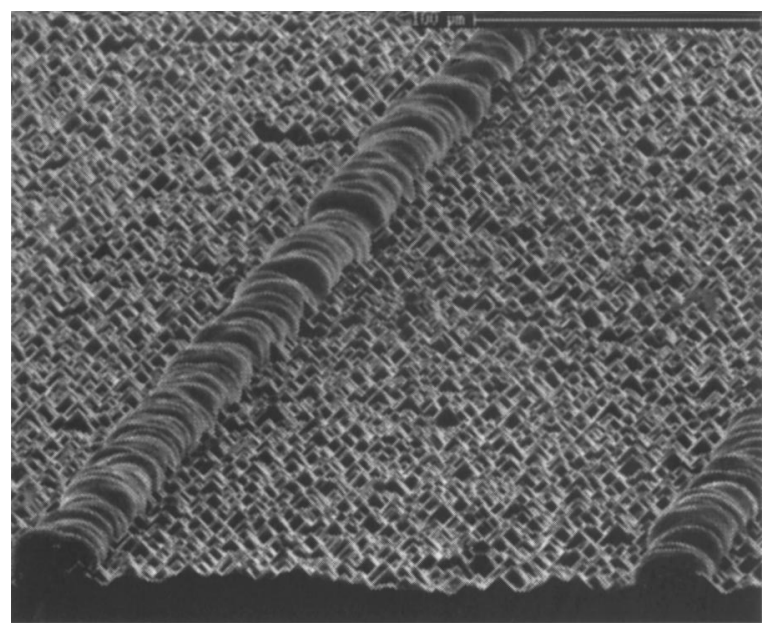

Fig. 4. Electroplated silver fingers on randomly textured silicon. The finger follows the textured surface.

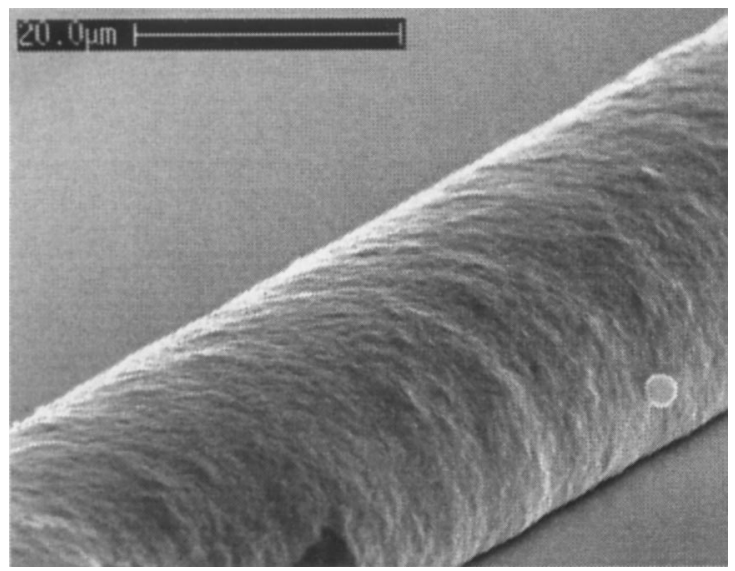

Fig. 5. A section of an electroplated silver finger on a polished silicon surface.

4. Light which is internally reflected at the glass air interface is trapped (this is not strictly true unless the coverage fraction is small, as discussed above).

Following the notation of Fig. 1, the total reflection and absorption loss due to the fingers is

$$
I_{\text {loss }}=\left[1-R_{\text {glass }}\right] \times\left[1-R_{\mathrm{Ag}} \times\left\{\int_{\alpha=\theta \mathrm{c} / 2}^{\alpha=90} \mathrm{~d} x+\int_{\alpha=0}^{\alpha=\theta \mathrm{c} / 2} f(2 \alpha) \mathrm{d} x\right\}\right] .
$$

Here $R_{\text {glass }}$ and $R_{\mathrm{Ag}}$ are the reflectance of glass and silver, respectively, $\alpha$ is the angle of the finger tangent from horizontal and $f(2 \alpha)$ is the function relating the partial internal 
Table 1

Theoretical calculations of reflection and absorption

\begin{tabular}{llllll}
\hline$n_{i}$ & $I_{\text {trap }}(\%)$ & $I_{\text {ref1 }}(\%)$ & $R_{\text {glass }}(\%)$ & $I_{\text {absorbed }}(\%)$ & $\begin{array}{c}\text { Effective shading } \\
\text { fraction }\left(I_{\text {loss }}\right)(\%)\end{array}$ \\
\hline 1.47 & 61.6 & 31.9 & 3.6 & 2.9 & 34.8 \\
1.48 & 61.9 & 31.5 & 3.8 & 2.9 & 34.4 \\
1.49 & 62.1 & 31.2 & 3.9 & 2.9 & 34.1 \\
1.5 & 62.3 & 30.9 & 4.0 & 2.9 & 33.8 \\
1.51 & 62.5 & 30.5 & 4.1 & 2.9 & 33.4 \\
\hline
\end{tabular}

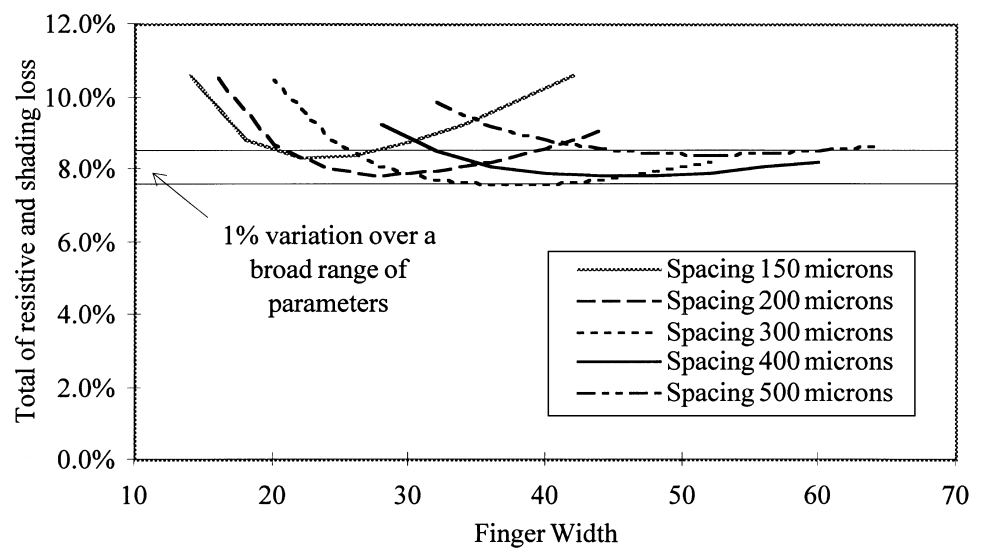

Fig. 6. Total loss associated with a silver electroplated front contact design as a function of finger width and spacing. The cell is $20 \mathrm{~cm}^{2}$ and the concentration is 30 suns.

reflection fraction to the incident angle (which is $2 \alpha$ ). This function, $f(2 \alpha)$, is a rearrangement of Fresnel's equations which results in a difficult analytical expression. The integration is achieved using Maple, a symbolic mathematics application, using numeric integration.

The results of the numerical calculations are included in Table 1 for glasses of different refractive index. The glass used at ANU has a refractive index of 1.511. They are in approximate agreement with the experimental results. The slight absorption in the encapsulant package $(<1 \%)$ would decrease the measured reflectance. Adding this into the theoretical calculations would slightly improve the fit between experiment and theory but as the absorption in the encapsulant package is at the limit of resolution of the spectrophotometer, the result would be of questionable value.

The experimentally determined effective width was used to find the optimum finger width and spacing. These calculations indicate that for silver-plated fingers on a planar surface, the total loss is only a weak function of finger spacing (see Fig. 6). Further, close to the optimum, the total loss is a rather weak function of crosssectional area due to the trade off between shading and finger resistance (see Fig. 6). 
Table 2

Primary losses associated with the metal contacts for a $20 \mathrm{~cm}^{2}$ cell operating at $3 \mathrm{~W} / \mathrm{cm}^{2}$. The assumed $V_{\text {oc }}$ is $750 \mathrm{mV}$ and $J_{\mathrm{sc}}$ is $40.4 \mathrm{~mA} / \mathrm{cm}^{2} /$ sun, minus shading loss. The analysis excludes contact recombination.

\begin{tabular}{lllllll}
\hline Cell type & $\begin{array}{l}\text { Finger } \\
\text { spacing } \\
\mu \mathrm{m}\end{array}$ & $\begin{array}{l}\text { Spreading } \\
\text { Loss }^{\mathrm{a}}(\%)\end{array}$ & $\begin{array}{l}\text { Effective } \\
\text { shading } \\
(\%)\end{array}$ & $\begin{array}{l}\text { Finger } \\
\text { resistance } \\
(\%)\end{array}$ & $\begin{array}{l}\text { Back metal } \\
\text { resistance } \\
(\%)\end{array}$ & Total (\%) \\
\hline Photolithographic & 200 & 0.4 & 4.5 & 2.2 & 0.7 & 7.8 \\
Front contact & 300 & 0.9 & 3.8 & 2.0 & 0.7 & 7.4 \\
Buried grid & 400 & 1.7 & 3.5 & 1.8 & 0.7 & 7.7 \\
Back contact & 400 & 1.7 & 4.0 & 0.7 & 0.7 & 7.1 \\
\hline
\end{tabular}

${ }^{\text {a }}$ For cells with a sheet emitter this is the emitter spreading resistance and for point or line diffused cells it is the loss associated with minority carrier flow and current crowding.

${ }^{\mathrm{b}}$ aspect ratio of 3 to 1 .

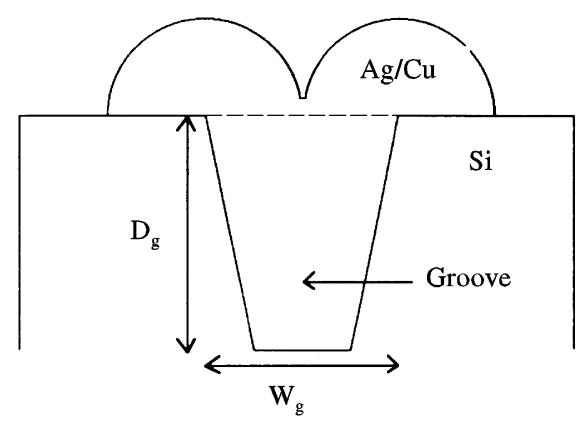

Fig. 7. Assumed cross-section for buried contacts.

Thus the spacing can be varied over a wide range to minimise the spreading, contact and recombination losses, which will be dependent on the processing technology.

A comparison with buried grid cells [2] and back contact cells [3] was performed based on the optimised parameters. Table 2 compares losses in front contact, rear interdigitated contact and buried grid cells. Contact recombination losses are not calculated but would be significantly less for the front contact and rear interdigitated contact designs than the buried grid design because of the lower contact area. A rear silver plating thickness of $5 \mu \mathrm{m}$ is assumed as thicker layers have been observed to delaminate during annealing steps. In each case the contact resistance is small if a resistance below $2 \times 10^{-5} \Omega / \mathrm{cm}^{2}$ is assumed.

For buried contact cells, the loss breakdown is almost identical to the electroplated fingers on a planar surface. Fig. 7 shows the buried contact cross section assumed in this study. This shape is based on the assumption that the plating proceeds isotropically from all areas of exposed silicon. This is generally a good assumption for modern plating solutions, when operated in the manner recommended by the manufacturer. In any case, if this assumption is broken, the plating in the groove would tend to suffer, resulting in a higher series resistance, without affecting the shading loss. In this study 
a groove cross-sectional area of $0.75 \times W_{\mathrm{g}} \times D_{\mathrm{g}}$ and a groove width of $25 \mu \mathrm{m}$ are assumed. This would probably be a best case for a production cell. An aspect ratio of 3 is assumed as this seems the highest likely aspect ratio achievable in production, based on the latest published technology [2], although current best practice is a commercial secret.

The resistive loss in a rear interdigitated finger pattern is significantly larger than the loss in a standard finger pattern because an interdigitated finger pattern requires a bus bar for each polarity, whereas a standard front grid pattern can use two bus bars of the same polarity. This halves the finger length and cuts the finger resistance by a factor of four. These calculations show that the loss associated with interdigitated rear contacts on large area substrates is comparable to the sum of the loss due to finger resistance and shading in front contact designs. Rear contact cells made on high resistivity wafers have an additional loss associated with the need to transport both electrons and holes to the rear surface. For this reason rear contact cells must be thin, which reduces light absorption by the solar cell.

It should be further noted that as calculated in Ref. [1], if the fingers are parallel to the incoming light, the effective width decreases with elevation angle. Thus in linear concentrators the net reflection will be reduced if the finger direction is perpendicular to the receiver axis because a large portion of the light striking the receiver is not perpendicular to the cell surface.

\section{Conclusions}

It is demonstrated that the optical width of an electroplated silver front grid is about one third of its geometric width. This is in agreement with the theoretical calculations and experimental measurements (using a different technique) presented in a previous study [1]. This leads to a need for recalculation of the optimum finger design of solar cells. The effective width has a slight dependence on coverage fraction due to the increased probability of trapped light striking a second finger and being coupled out.

A comparison of the optical performance of conventional front contact cells with rear point contact cells indicates that at 30 suns a rear interdigitated finger contact design has a power loss similar to the standard front contact design unless a complex rear mounting scheme (similar to 300 sun cells [8]) is used. Buried grid cells have a similar optical loss to cells with electroplated fingers on a planar surface, although recombination losses will be larger.

A more detailed analysis could include the effects of finite substrate resistivity and three dimensional transport effects, as well as the loss of current due to cell thickness or recombination loss if the cell is not thinned.

\section{Acknowledgements}

We gratefully acknowledge the Research School of Chemistry Inorganic Electrochemistry Group, and the Electron Microscopy Unit at ANU for the use of their 
facilities. This work was partially funded by the Australian Research Council and Solahart Industries.

\section{References}

[1] A. Blakers, J. Appl. Phys. 71 (10) 15 (1992) 5237.

[2] F.Z. Zhang, S.R. Wenham, M.A. Green, IEEE Trans. Electron. Dev. 42 (1) (1995) 144.

[3] R.A. Sinton, R.M. Swanson, IEEE Trans. Electron. Dev. 37 (2) (1990) 348.

[4] M.J. O'Neill, A.J. McDanal, The 25 kilo watt solar row: a building block for utility-scale concentrator systems, 25th IEEE PVSC, May 1996, Washington D.C., pp. 1529-1532.

[5] G. Sala et. al., The $480 \mathrm{~kW}$ peak EUCLIDES ${ }^{\mathrm{TM}}$ concentrator power plant using parabolic troughs, second WCEPSEC, Vienna, July 1998, in press.

[6] J. Smeltink, A.W. Blakers, The ANU PV/Trough concentrator system, second WCEPSEC, Vienna, July 1998, in press.

[7] D. Stellbogen, H.-D. Mohring, G. Schumm, Deflecting cell cover for parabolic mirror PV concentrators, 13th EPSEC, Nice, October 1995, pp. 1479-1482.

[8] R.A. Sinton, P.J. Verlinden, R.A. Crane, R.M. Swanson, Development of Manufacturing Capability for High-Concentration, High-Efficiency Silicon Solar Cells, Contractor Report Sand96-2403, USDOE. 